\title{
Dual CD19 and CD123 targeting prevents antigen-loss relapses after CD19-directed immunotherapies
}

\author{
Marco Ruella, ${ }^{1,2,3}$ David M. Barrett, ${ }^{4}$ Saad S. Kenderian, ${ }^{1,5}$ Olga Shestova, ${ }^{1}$ Ted J. Hofmann, ${ }^{4}$ Jessica Perazzelli, ${ }^{4}$ \\ Michael Klichinsky, ${ }^{1}$ Vania Aikawa, ${ }^{2}$ Farzana Nazimuddin, ${ }^{1}$ Miroslaw Kozlowski, John Scholler, ${ }^{1}$ Simon F. Lacey, ${ }^{1,2}$ \\ Jan J. Melenhorst,, ${ }^{1,2}$ Jennifer J.D. Morrissette, ${ }^{2}$ David A. Christian, ${ }^{6}$ Christopher A. Hunter, ${ }^{6}$ Michael Kalos, ${ }^{1}$ \\ David L. Porter, ${ }^{3,7}$ Carl H. June, ${ }^{1,2,3}$ Stephan A. Grupp, ${ }^{4}$ and Saar Gill1,3,7 \\ 'Center for Cellular Immunotherapies and ²Department of Pathology and Laboratory Medicine, Perelman School of Medicine at the University of Pennsylvania, Philadelphia, Pennsylvania, USA. \\ ${ }^{3}$ Abramson Cancer Center at the University of Pennsylvania, Philadelphia, Pennsylvania, USA. ${ }^{4}$ Division of Oncology, The Children's Hospital of Philadelphia, Philadelphia, Pennsylvania, USA. \\ ${ }^{5}$ Division of Hematology, Department of Internal Medicine, Mayo Clinic, Rochester, Minnesota, USA. ${ }^{6}$ Department of Pathobiology, School of Veterinary Medicine at the University of Pennsylvania, \\ Philadelphia, Pennsylvania, USA. 'Division of Hematology-Oncology, Department of Medicine, Perelman School of Medicine at the University of Pennsylvania, Philadelphia, Pennsylvania, USA.
}

\begin{abstract}
Potent CD19-directed immunotherapies, such as chimeric antigen receptor T cells (CART) and blinatumomab, have drastically changed the outcome of patients with relapsed/refractory B cell acute lymphoblastic leukemia (B-ALL). However, CD19negative relapses have emerged as a major problem that is observed in approximately $30 \%$ of treated patients. Developing approaches to preventing and treating antigen-loss escapes would therefore represent a vertical advance in the field. Here, we found that in primary patient samples, the IL-3 receptor $\alpha$ chain CD123 was highly expressed on leukemia-initiating cells and CD19-negative blasts in bulk B-ALL at baseline and at relapse after CART19 administration. Using intravital imaging in an antigen-loss CD19-negative relapse xenograft model, we determined that CART123, but not CART19, recognized leukemic blasts, established protracted synapses, and eradicated CD19-negative leukemia, leading to prolonged survival. Furthermore, combining CART19 and CART123 prevented antigen-loss relapses in xenograft models. Finally, we devised a dual CARexpressing construct that combined CD19- and CD123-mediated T cell activation and demonstrated that it provides superior in vivo activity against B-ALL compared with single-expressing CART or pooled combination CART. In conclusion, these findings indicate that targeting CD19 and CD123 on leukemic blasts represents an effective strategy for treating and preventing antigen-loss relapses occurring after CD19-directed therapies
\end{abstract}

\section{Introduction}

Chemo-refractory or relapsing $(\mathrm{R} / \mathrm{R}) \mathrm{B}$ cell acute lymphoblastic leukemia (B-ALL) is associated with a poor prognosis (1-4) yet remains responsive to targeted immune-based therapy. In particular, anti-CD19 chimeric antigen receptor T cells (CART19) and bispecific anti-CD19/CD3 antibodies (blinatumomab) generate unprecedented complete response rates in this patient population (5-9). Both approaches redirect autologous $\mathrm{T}$ cells to recognize CD19-expressing cells. Blinatumomab infusion recruits endogenous T cells to engage the tumor, while CART19 cells are genetically modified ex vivo to express an anti-CD19 single-chain variable fragment $(\mathrm{scFv})$ fused to the $\mathrm{T}$ cell receptor signaling molecule $\mathrm{CD} 3-\zeta$ with built-in costimulatory domains and then reinfused into the patient (10). We recently showed that approximately $90 \%$ of patients with R/R B-ALL treated with CART19

Authorship note: M. Ruella, D.M. Barrett, S.A. Grupp, and S. Gill contributed equally to this work.

Conflict of interest: The authors work under a research collaboration involving the University of Pennsylvania and the Novartis Institutes of Biomedical Research Inc. M. Ruella, S.S. Kenderian, J. Scholler, S.F. Lacey, J.J. Melenhorst, J.J.D. Morrissette, M. Kalos, D.L. Porter, C.H. June, and S. Gill are inventors of intellectual property licensed by the University of Pennsylvania to Novartis.

Submitted: February 29, 2016; Accepted: July 14, 2016.

Reference information: / Clin Invest. 2016;126(10):3814-3826. doi:10.1172/JCI87366.
(CTL019) achieved complete remission (CR) (6), and similar results have been found by other groups. $(7,8,11)$ Despite high initial response rates, some patients relapse, and up to $30 \%$ of relapses after blinatumomab and $60 \%$ after CART19 are characterized by the loss of CD19 antigen, rendering the malignant cells invisible to CD19-specific immunotherapies $(5,6,12,13)$. This observation simultaneously illustrates the power and the shortcoming of antigen-specific immunotherapy.

CD19 is a prototypic B cell lineage marker that is widely expressed during B cell development. CD19 plays an important role in B cell biology, as CD19-deficient B cells exhibit selective growth disadvantage (14). Thus, the absence of CD19 is a very unusual finding in B-ALL and was reported in only rare cases prior to the advent of potent CD19-directed immunotherapies (15-17). Our group recently described one mechanism for CD19 loss, finding that in some patients, alternative exon splicing of CD19 leads to loss of the CD19 epitope that is recognized by CART19 (18), prompting us to search for additional mechanisms that would allow B-ALL cells to escape anti-CD19 immunotherapy. We hypothesized that the existence of immature CD19 negative B-ALL precursors could predispose to antigen-loss escape.

The IL-3 receptor $\alpha$ chain (CD123) is expressed in several hematologic neoplasms, including acute myeloid leukemia (AML), plasmacytoid dendritic cell neoplasm (19), hairy cell leukemia 

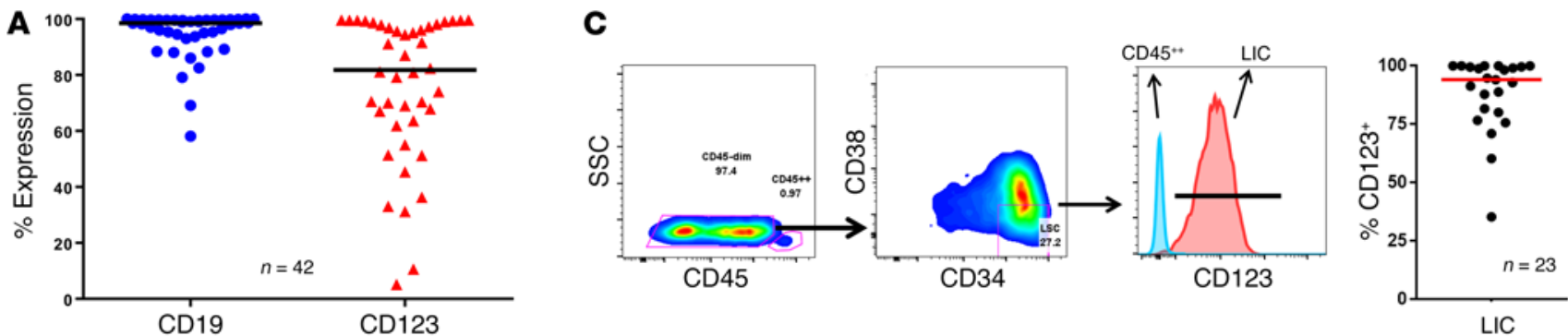

B

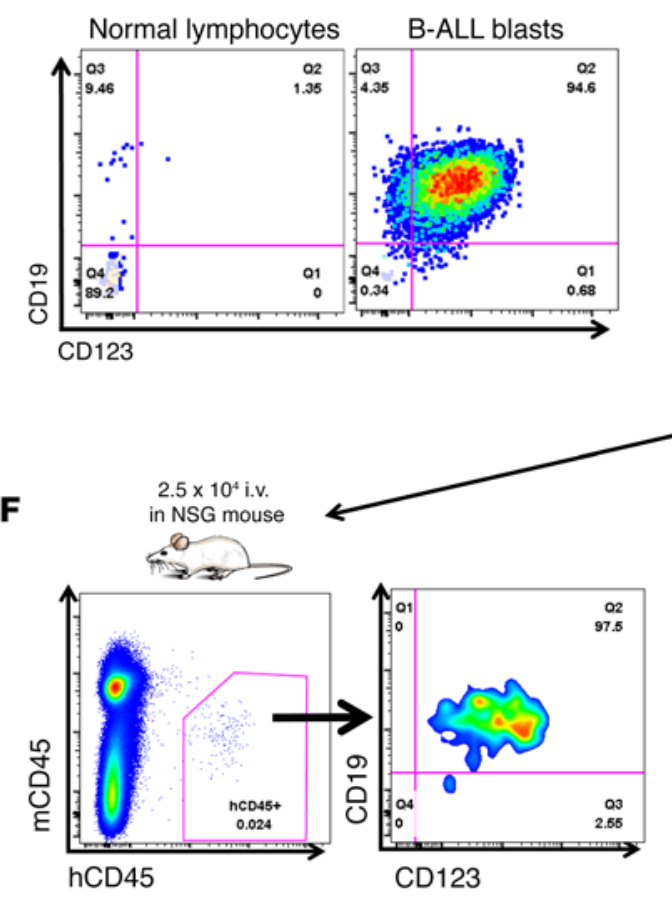

D
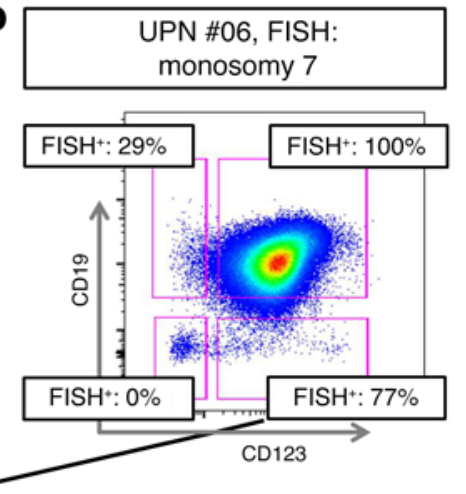

$\mathbf{E}$

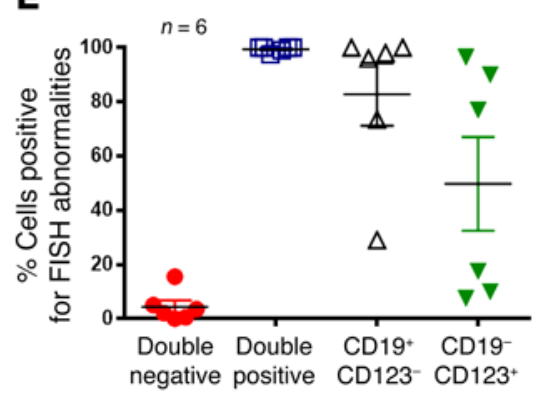

G $\square$ Isotype control $\square$ Baseline $\square$ Relapse after CTL019
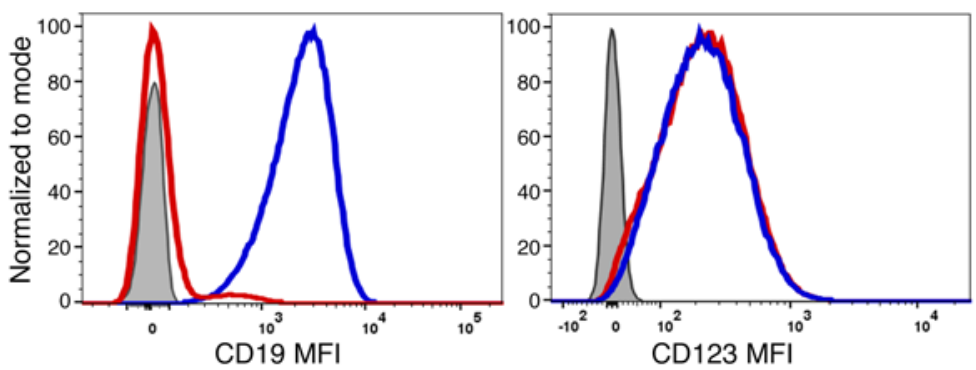

Figure 1. CD123 is highly expressed in B-ALL, including the LIC and the CD19-negative relapses occurring after CD19-targeted immunotherapies. (A) High expression of CD123 in 42 R/R B-ALL samples (gated on blasts: SSC ${ }^{10}$, single, live, CD45 ${ }^{\text {dim/neg) }}$. (B) CD123 and CD19 are typically coexpressed in B-ALL blasts, although minor CD19-negative and CD123-negative blasts can be detected at very low frequencies. (C) CD123 is expressed in the LIC compartment (red), defined as CD34+CD38- blasts (blue represents control CD45++ cells). Rlght panel shows expression of CD123 in the ALL LIC of 23 B-ALL patients. (D) B-ALL

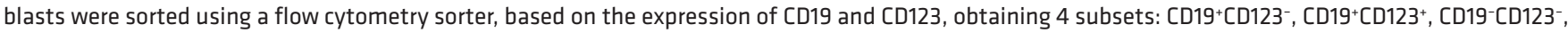
and $\mathrm{CD} 19^{-} \mathrm{CD} 123^{+}$. These 4 subsets were highly pure and were analyzed by FISH for their specific genetic marker. Boxes indicate percentage of FISH-positive cells in each subset. Importantly, CD19-CD123+ blasts were positive in most cases. (E) The sorting experiment was repeated in a total of 6 B-ALL samples with different FISH abnormalities, and results were consistent. (F) Injection of sorted CD19-negative CD123-positive leukemic subpopulations into NSG mice led to reconstitution of the original B-ALL phenotype (BM, day 120), confirming this population's LIC function (graph representative of 2 independent experiments each with 2 mice per group). (C) CD123 expression (MFI, mean fluorescence intensity) is maintained in CD19-negative B-ALL relapses occurring after CART19 (CTL019) treatment; representative case is shown. Gating is based on isotype control.

(20), and Hodgkin lymphoma (21-24). Unlike lineage-associated surface antigens such as CD33 (myeloid) or CD19 (B lymphoid), CD123 is hierarchically expressed on hematopoietic progenitor cells, and in AML, CD123 is expressed on leukemic stem cells that are involved in resistance to chemotherapy and relapse after initial treatment $(25,26)$. Importantly, CD123 has also been reported to be broadly expressed in both pediatric and adult ALL (27-30). Due to these characteristics, multiple modalities to target CD123 in hematological neoplasms have been developed, such as the IL-3 diphtheria toxin fusion protein (SL-401, DT388IL3) $(31,32)$, unconjugated anti-CD123 monoclonal antibodies (CSL-360, CSL362) (33), antibody-drug conjugates (34), bispecific antibodies
$(35,36)$ or CD3Fv-IL-3 fusion constructs $(37)$, and more recently, CART123 (22, 38-40). Our group found that targeting CART123 in preclinical xenograft models engenders deep and durable responses in human primary AML xenografts and can establish an antileukemia T cell memory (38). Several phase 1 clinical trials testing anti-CD123 CART are enrolling patients at the time of this writing, including one such trial at the University of Pennsylvania (ClinicalTrials.gov NCT02623582). Here we demonstrate in B-ALL the existence of rare CD19-negative malignant cells expressing CD123 at baseline and show that these cells can be responsible for relapse. We also find that CD123 is retained in B-ALL patients relapsing after CART19 therapy. Finally, we show that by combin- 


\section{Table 1. Characteristics of B-ALL samples}

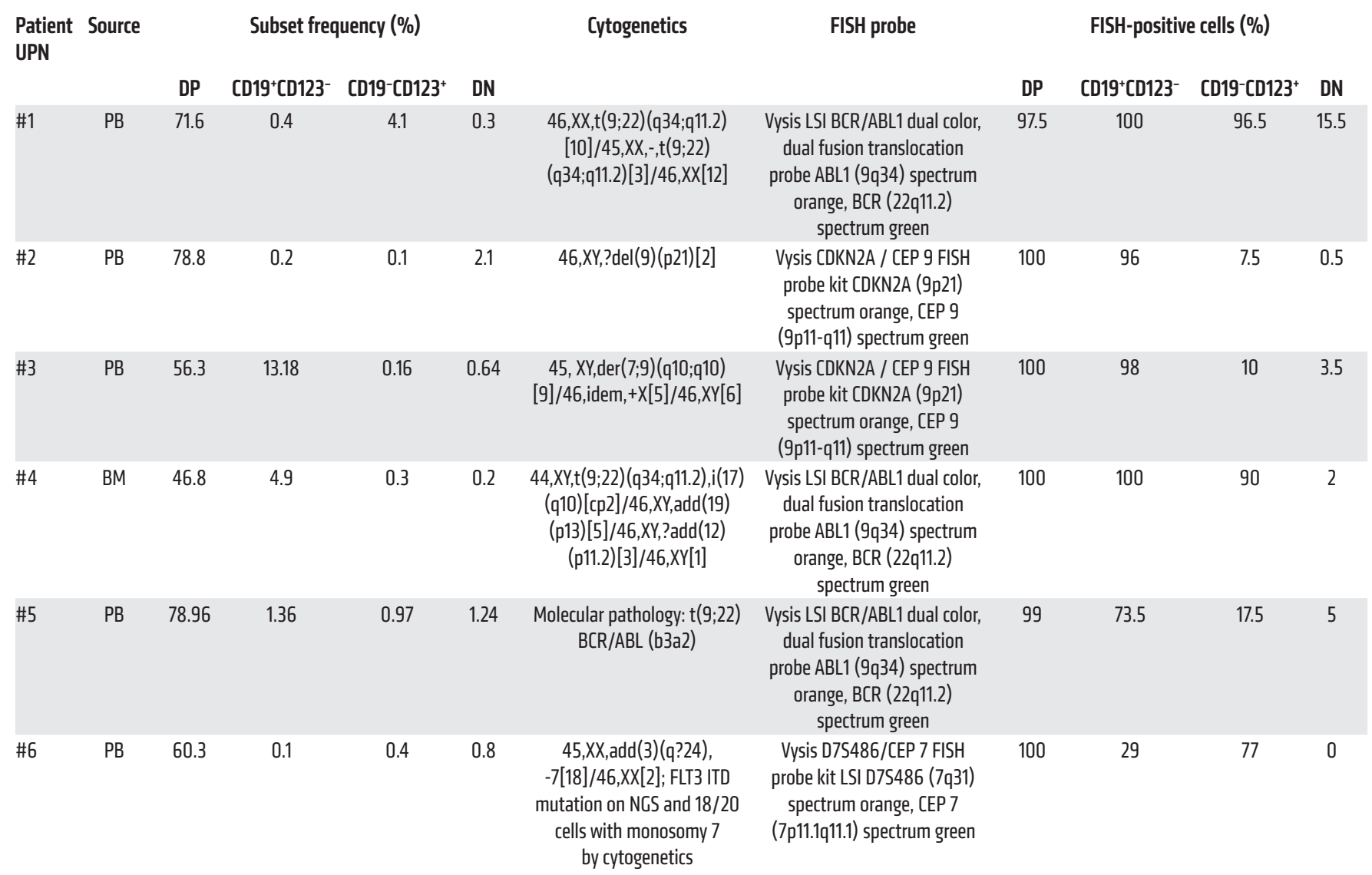

Samples were sorted for 4 cell subsets based on the expression of CD19 and CD123. FISH was then performed in each subset to investigate the presence of the chromosomal abnormality specific for that patient's leukemia. DP, double positive; DN, double negative.

ing CART123 cells with the current CART19 approach, we can treat and prevent CD19-loss relapses in B-ALL xenografts, thus paving the way to combination immunotherapy in the clinic.

\section{Results}

CD123 is expressed in B-ALL within both bulk and the leukemiainitiating cell populations and is retained in post-CTLO19 CD19negative relapses. In order to evaluate the expression of CD123 in B-ALL, we analyzed 42 samples from adult and pediatric ALL patients, including 14 subjects enrolled in our current CTL019 clinical trials. As shown in Figure 1, A and B, and Supplemental Figure 1A (supplemental material available online with this article; doi:10.1172/JCI87336DS1), CD123 is robustly and homogeneously expressed on the surface of most ALL blasts, representing a potential candidate for targeted therapy. Moreover, CD123 is also found to be expressed in leukemia-initiating cells (LIC), both as identified by phenotype $\left(\mathrm{CD} 34^{+} \mathrm{CD} 38^{-}\right)$(Figure $\left.1 \mathrm{C}\right)$ and by their ability to engraft in NOD-SCID $\gamma$ chain-deficient (NSG) mice, giving rise to overt leukemia (Supplemental Figure 1B). Small subpopulations of CD19-negative CD123-positive CD45 ${ }^{\mathrm{dim}}$ cells could be identified in B-ALL patients (Figure 1D), and they have a LIC-like phenotype $\left(\mathrm{CD} 34^{+} \mathrm{CD} 38^{-}\right)$(Supplemental Figure $\left.1 \mathrm{C}\right)$. We hypothesized that these cells could be clonal and therefore might contribute to antigen-loss relapses (41). To demonstrate that some CD19-negative cells in B-ALL arise from the malignant clone, we selected primary B-ALL specimens with an informative cytogenetic profile from our institutional repository and sorted $\mathrm{CD}^{-} 9^{-} \mathrm{CD} 123^{-}, \mathrm{CD}^{-} 9^{-} \mathrm{CD} 123^{+}, \mathrm{CD}_{19}{ }^{+} \mathrm{CD} 123^{-}$, and $\mathrm{CD} 19^{+} \mathrm{CD} 123^{+}$ cells from the B-ALL blast population (CD45 ${ }^{\mathrm{dim}}, \mathrm{SSC}^{\mathrm{lo}}$, where SSC indicates side scatter; gating strategy shown in Supplemental Figure 2, A and B), followed by FISH for the informative marker (Figure 1D). We found that $\mathrm{CD}^{-} \mathrm{C}^{-} \mathrm{CD} 123^{+}$blasts carried the diseaseassociated genetic aberration, albeit at a lower frequency than the CD19+ blasts, whereas $\mathrm{CD}^{+} 9^{-} \mathrm{CD} 123^{-}$cells did not (Figure 1D, Supplemental Figure 2, A-C, and Table 1). In 6 of the 6 samples (100\%) we tested, the CD19- CD123+ blasts harbored some level of the disease-associated genetic aberration (Figure 1E). This finding indicates that targeting CD19 alone could, in some cases, lead to a subclonal relapse derived from preexisting CD19-CD123+ cells. Furthermore, injection of sorted CD19-negative CD123-positive subpopulations into NSG mice led to reconstitution of the original B-ALL phenotype, confirming this population's leukemic stem cell function (Figure 1F). Finally, the expression of CD123 was also evaluated in archived samples of B-ALL patients who relapsed after CTL019 with loss of CD19. Notably, in contrast to the complete loss of CD19, the majority of patients maintained CD123 


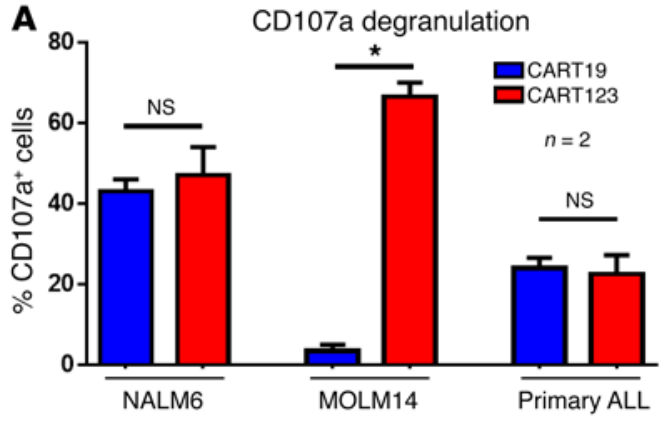

C

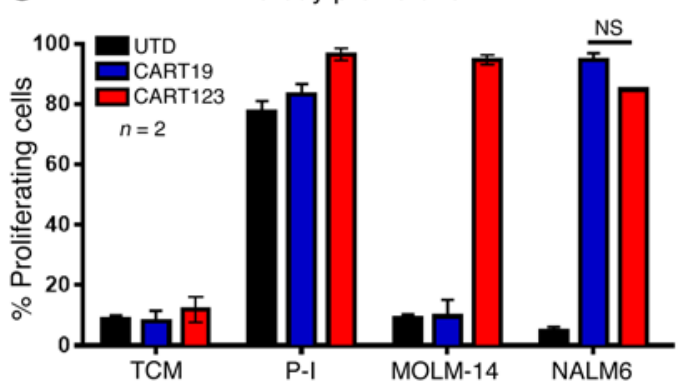

B

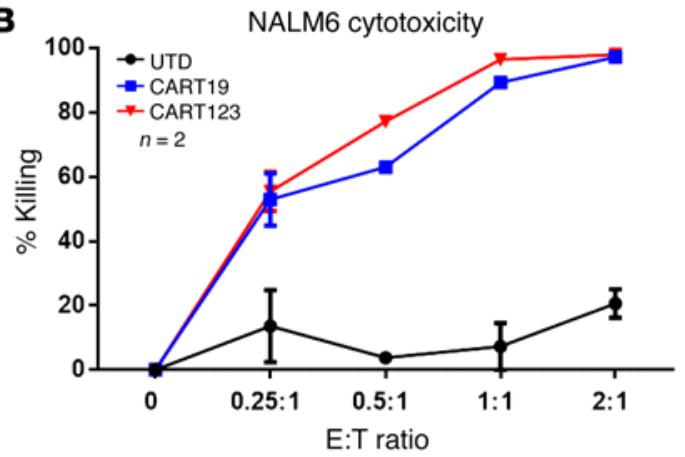

E

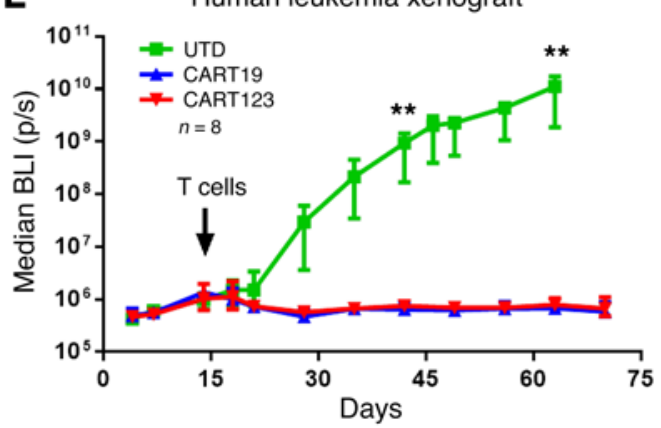

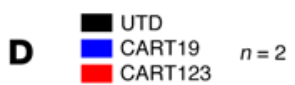

CART123
IFN- $\gamma$

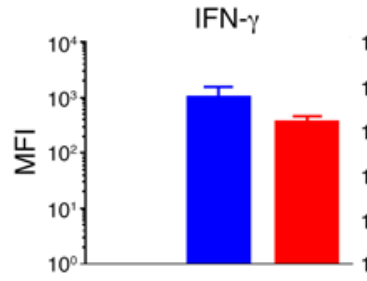

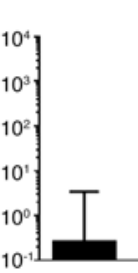

IL-2

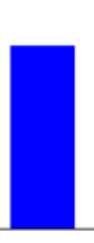

Cytokine production
GM-CSF

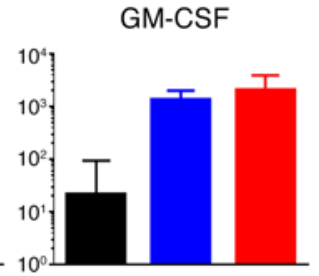

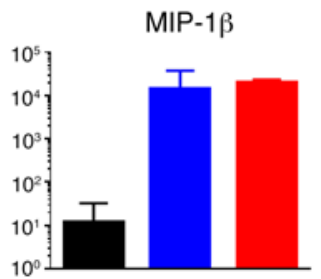

Figure 2. CART123 exerts potent antileukemia activity in vitro and in vivo. (A) CART123 and CART19 cells were cocultured with B-ALL blasts (the cell line NALM6 or primary blasts, CD19+, CD123+) or a CD19-negative cell line (the CD123+ AML cell line MOLM-14) for 4 to 6 hours. Both CART123 and CART19 showed similar expression of CD107a, a marker of degranulation/activation when cocultured with NALM6 and primary ALL, while only CART123 recognized the CD123+ and CD19- cell line MOLM-14. (B) Cytotoxicity at 24 hours of CART123, CART19, or control T cells (UTD) when cocultured at different E:T ratios with NALM6. Increasing concentration of either CART123 or CART19 led to similar levels of killing of NALM6, while no killing was observed in the control group (UTD). (C) CFSE-stained CART123, CART19, or UTD was cocultured with medium only (TCM), PMA-ionomycin (nonspecific stimulus), MOLM-14 $\left(\mathrm{CD} 123^{+}, \mathrm{CD}^{-} 9^{-}\right)$, or B-ALL blasts (NALM6 or primary blasts, CD19+, CD123+) for 5 days at a 1:1 E:T ratio; then CFSE dilution was analyzed by flow cytometry. High proliferation was observed in both CART123 and CART19 when cocultured with B-ALL blasts, but not in controls (UTD). As a control, CART123 also proliferated with MOLM-14. TCM, tissue culture medium. (D) Cytokine production by CART123, CART19, or UTD incubated with NALM6 for 3 days at 1:1 E:T ratio was analyzed in the culture supernatants. Significant production of several cytokines is noted in CART 19 and CART 123 groups, but not in UTD. (E) Luciferase-positive primary B-ALL blasts (patient UPN\#11) were injected in NSG mice, and after 14 days, mice were randomized based on tumor burden to receive either CART123, CART19, or control T cells (UTD). Mice receiving CART123 or CART19, but not UTD, showed quick leukemia remission that was maintained in the long term. All graphs are representative of at least 2 independent experiments. Student's $t$ test was used to compare 2 groups; in analysis where multiple groups were compared, 1-way ANOVA was performed with Holm-Šidák correction for multiple comparisons. When multiple groups at multiple time points/ratio were compared, Student's $t$ test or ANOVA for each time point/ratios was used. ${ }^{*} P<0.05 ;{ }^{*} P<0.01$.

expression at relapse (Figure 1G and Supplemental Figure 2D). These findings indicate that CD123 represents a potential therapeutic target in the setting of CD19-negative ALL occurring after CART19 or blinatumomab.

CART123 are active against human B-ALL in vitro and in vivo. Having previously generated CART123 for AML (38), we next evaluated their activity against B-ALL using the $\mathrm{CD} 19^{+} \mathrm{CD} 123^{+}$ B-ALL cell line NALM6 as well as primary B-ALL samples. CART123 and CART19 demonstrated equivalent degranulation, dose-dependent killing, proliferation, and cytokine produc- tion (Figure 2, A-D). These results support the potential role of CART123 in B-ALL. In order to confirm these data in an in vivo model, we utilized a primary ALL xenograft model (42). NSG mice engrafted with GFP/Luc ${ }^{+}$primary ALL blasts i.v. (patient UPN\#11, $\mathrm{CD} 45^{\mathrm{dim}}, \mathrm{CD}^{+} 9^{+}, \mathrm{CD}_{123^{+}}, \mathrm{CD}_{10}{ }^{+}, \mathrm{CD}_{4} 4^{+-}$) were randomized to receive CART19, CART123, or control untransduced $\mathrm{T}$ cells (UTD). Mice treated with control T cells succumbed quickly to disease (median survival, 75 days), while mice treated with either CART19 or CART123 showed tumor eradication and long-term survival (median survival not reached, $P<0.0001$ ) (Figure $2 \mathrm{E}$ and 
A
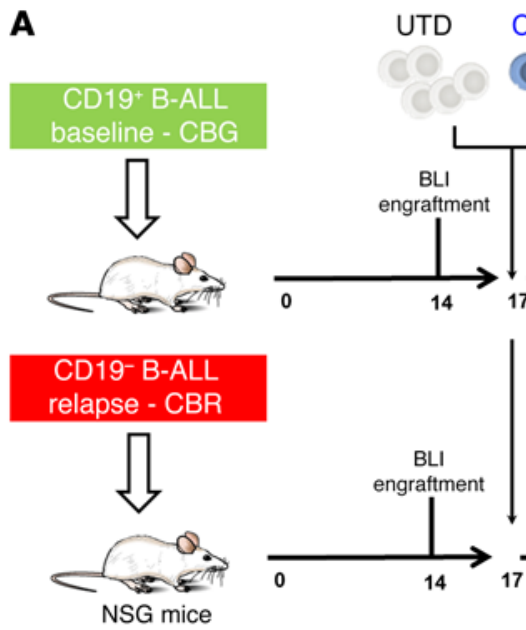

CART19

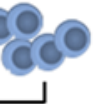

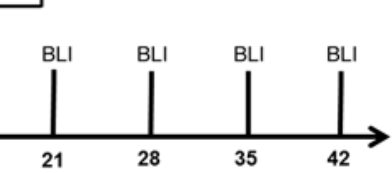

Leukemia burden

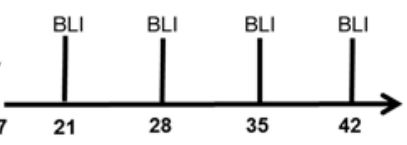

B
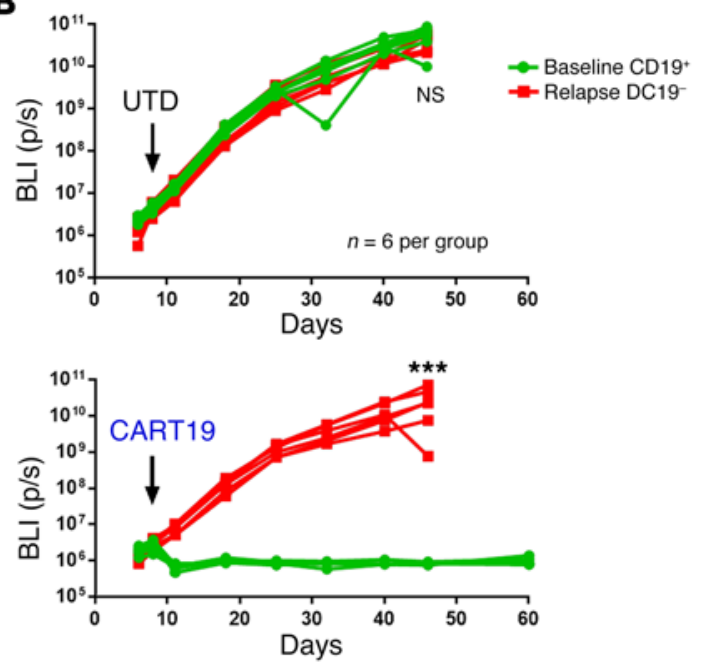

c

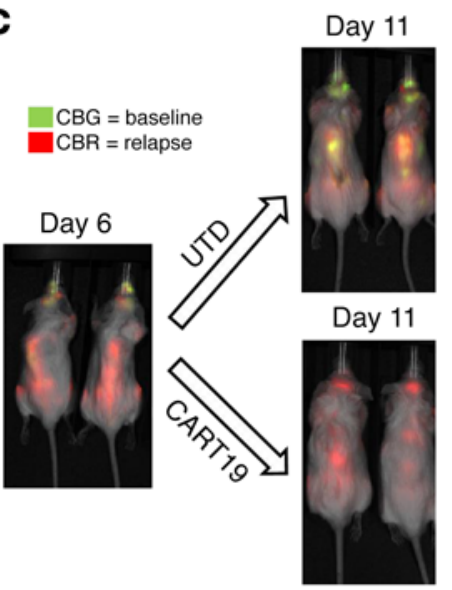

D

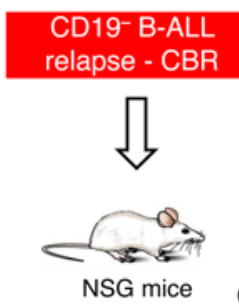

E

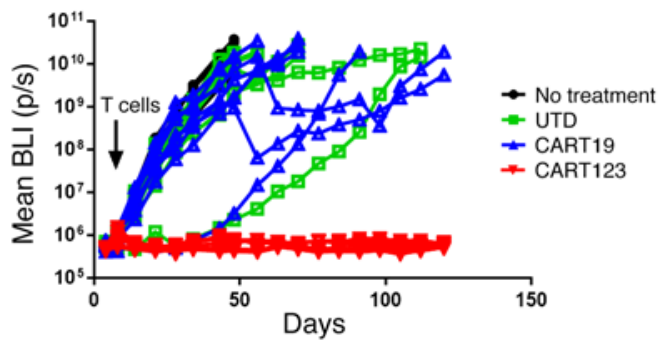

UTD CART19 CART123

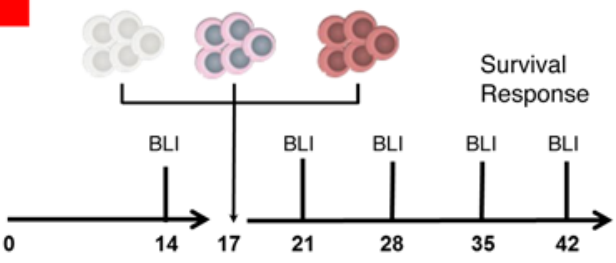

$\mathbf{F}$

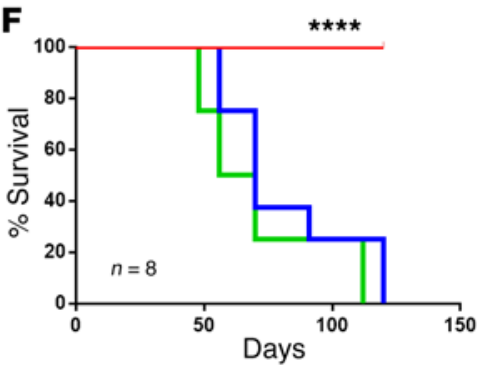

Figure 3. CART123, but not CART19, can successfully target CD19-negative B-ALL relapses. (A) A human xenograft model of CD19-negative relapse; experiment schematic. Two groups of NSG mice were respectively engrafted with B-ALL blasts (luciferase positive) originally obtained from the same patient (patient UPN\#09) at baseline and when the patient relapsed with a CD19-negative disease after CART19. At day 17, mice in each group were randomized to receive CART19 or control T cells (UTD). Leukemia burden was followed using bioluminescence (BLI). (B) In both groups (baseline and relapse), mice treated with UTD showed disease progression (top graph). CART19 are only capable of inducing responses in the group of mice engrafted with baseline disease, while no antileukemia effect is observed in the group engrafted with CD19-negative relapse disease (bottom graph). (C) In another in vivo model, baseline (CD19+, CBC luciferase ${ }^{+}$) and relapsed (CD19-, CBR luciferase+) blasts from the same patient were mixed 1:1 and injected in the same NSG mice. Mice were then treated with CART19 or UTD, and the 2 B-ALL populations (baseline vs. relapse) were followed by bioluminescence. In mice treated with UTD, both populations showed progression (CBG and CBR bioluminescence), while in CART19-treated mice, only the relapsed clone (CD19-negative) showed frank progression (CBR). (D) CART123 successfully eliminated CD19-negative B-ALL relapses in vivo. Experiment schematic: luciferase-positive primary CD19-negative relapsed B-ALL blasts were injected in NSG mice, and after 17 days, mice were randomized based on tumor burden to receive either CART123, CART19, control T cells (UTD), or no treatment. (E) Mice receiving CART123 but not CART19 or UTD showed quick leukemia remission that was maintained in the long term (>120 days), leading to a significant advantage in overall survival (F). All graphs arerepresentative of 2 independent experiments (6-8 mice per group). Student's $t$ test or ANOVA for each time point/ratio was used. Survival curves were compared using the log-rank test. ${ }^{* * *} P<0.001 ;{ }^{* * * *} P<0.0001$.

Supplemental Figure 3A). CART123 T cells significantly expanded in the peripheral blood $(\mathrm{PB})$ of the mice compared with control $\mathrm{T}$ cells and expressed high levels of CART123 (Supplemental Figure 3B). The antileukemia activity of CART123 was specific and based on the recognition of CD123 in the surface of the blasts, as when we engrafted mice with a rare $\mathrm{CD} 123^{-} \mathrm{CD} 19^{+}$leukemia from our repository (patient UPN\#12), only CART19 showed antileukemia activity, while CART123 had no effect as compared with control UTD $(P=0.0003)$ (Supplemental Figure 3, C and D).

CART123 but not CART19 are active in a preclinical model of antigen-loss relapse. In order to test new strategies for targeting CD19-negative relapses, we developed an vivo model of anti- 
A

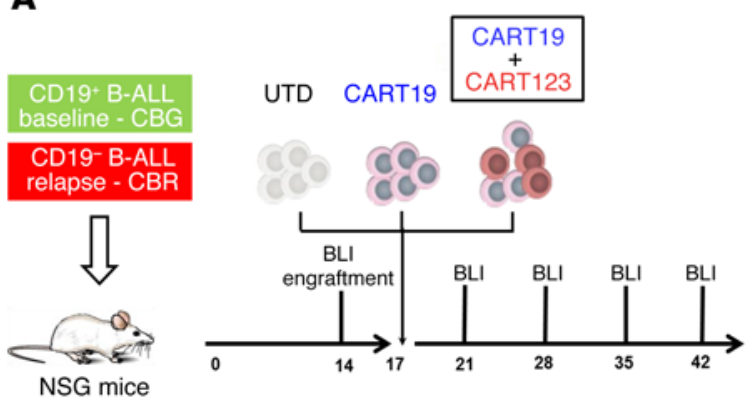

B
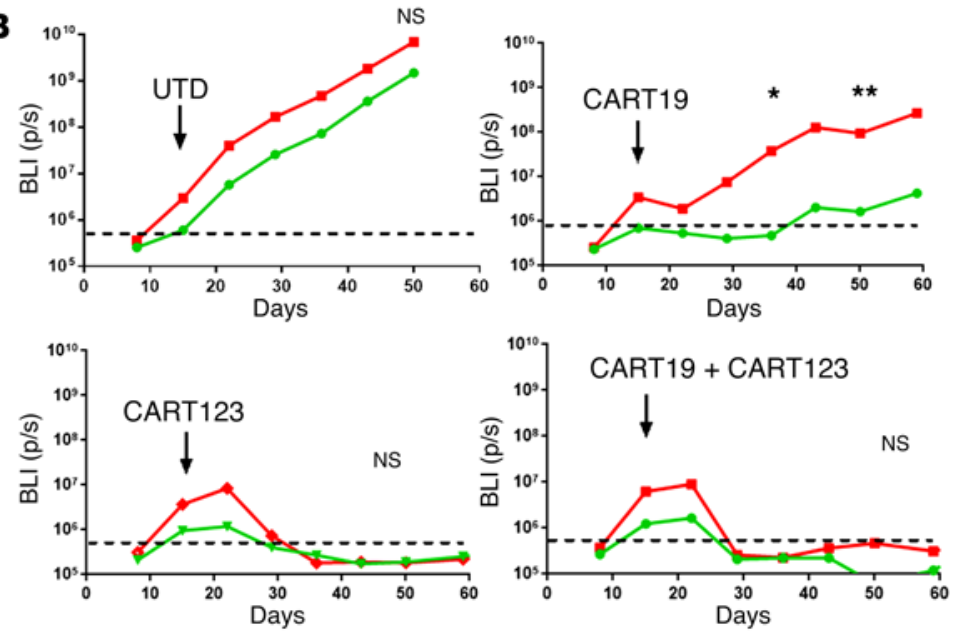

- Baseline CD19+ B-ALL -Relapse CD19- B-ALL $n=8$
C

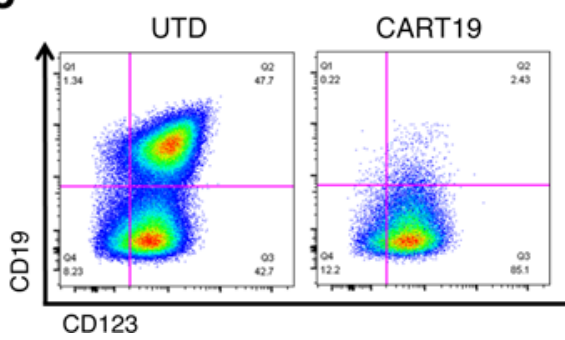

CART $19+$ CART123

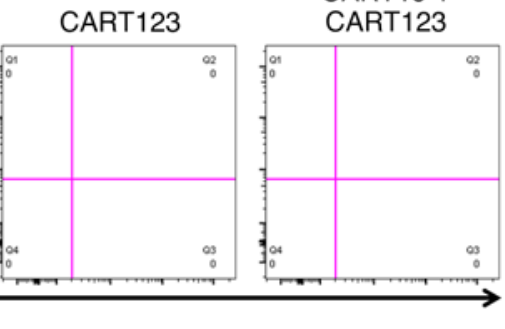

Figure 4. Preventing CD19-negative relapses using combination of CART19 and CART123. (A) Experiment schematic: NSG mice were engrafted with a 1:1 mixture of B-ALL blasts obtained from a patient (patient UPN\#09) at baseline when the disease was CD19+CD123+ (CBC luciferase ${ }^{+}$) and at relapse when the disease was CD19-CD123+ (CBR luciferase+). At day 17, mice were randomized to receive CART19, control T cells (UTD), or the 1:1 pooled combination of CART123 and CART19. (B) Mice treated with UTD showed progression of both CD19+ baseline ALL and CD19- relapsed ALL, while mice receiving CART19 showed progression with a CD19-negative disease. Only CART123 or the combination of CART123 and CART19 was able to prevent the onset of CD19- relapse. (C) Representative leukemia phenotype observed in the spleen of mice sacrificed at day 50 (gated on human SSC ${ }^{10}$, CD45 dim, live, single, GFP+ blasts): UTD-treated mice progress, with both $\mathrm{CD}_{19}{ }^{+}$and CD19- ALL, while CART19-treated mice showed clear CD19-negative progression. CART123 and the combination of CART123 and CART19 led to the clearance of both CD19+ and CD19- leukemia in most of the mice. Representative of 2 independent experiments. Student's $t$ test for each time point/ratio was used. ${ }^{*} P<0.05 ;{ }^{* *} P<0.01$.

gen-loss relapse. B cell blasts obtained from a patient (UPN\#09) enrolled in one of our CTLO19 clinical trials were collected at baseline (before CART19 therapy), when the disease was $\mathrm{CD}^{+} 9^{+}$, and $\mathrm{CD}_{123^{+}}$and at relapse after CART19, when the patient developed a CD19-negative disease (CD123 still expressed, Supplemental Figure 4A) (41). In a first set of experiments, baseline and relapsed blasts were transduced with luciferase and separately injected in NSG mice. Both groups of mice were randomized to receive CART19 or control T cells (UTD) (Figure 3A). As expected, UTD failed to control disease in either group, whereas CART19 eradicated the baseline $\mathrm{CD} 19^{+}$ALL while failing to control CD19disease $(P=0.0006)$ (Figure 3B). To establish whether activation of CART19 by CD19+ ALL could lead to bystander killing of CD19blasts, NSG mice engrafted with a 1:1 mixture of $\mathrm{CD} 19^{+}$baseline and $\mathrm{CD}_{19}$ relapsed ALL were randomized to receive CART19 or control $\mathrm{T}$ cells. Tumor burden was monitored with bioluminescence imaging that could discriminate between $\mathrm{CD}_{1} 9^{+}$(click beetle green [CBG]) and CD19- (click beetle red [CBR]) leukemia growth in vivo. As shown in Figure 3C, in mice receiving UTD, both $\mathrm{CD} 19^{+}$(green) and $\mathrm{CD}_{19}^{-}$(red) leukemias present at day 6 were similarly increased at day 11, while in mice treated with CART19, the baseline disease (green) was completely cleared, while the relapsed disease (red) showed progression. Thus, we confirmed in a tractable in vivo xenograft model our clinical observations that CART19 are completely ineffective in eradicating CD19-negative relapsed disease, with no evidence of bystander killing.

We next used this CD19-negative relapsed B-ALL model to evaluate the role of CART123 in the treatment of antigen-loss relapses. We initially tested the ability of CART123 to be specifically activated by CD19-negative ALL blasts (Supplemental Figure 4B). Consequently, primary CD19-negative blasts (CBR positive) were injected into NSG mice (Figure 3D) and mice were randomized to receive CART19, CART123, or control T cells (UTD). CART19 and control T cells lacked antitumor activity, while CART123 led to eradication of the disease and long-term survival of these mice (median survival not reached vs. 70 days, $P<0.0001$ compared with CART19) (Figure 3, E and F). No CD123negative relapses were observed in this model at up to 3 months of follow-up. These observations establish the role of CART123 in a preclinical model of primary B-ALL after failure of CART19.

In order to understand the differential behavior of CART19 and CART123 in this in vivo model at a single-cell level, we performed a series of experiments injecting a mixture of differentially labeled CART19 (CellTrace Violet, blue) and CART123 (Cell- 
A

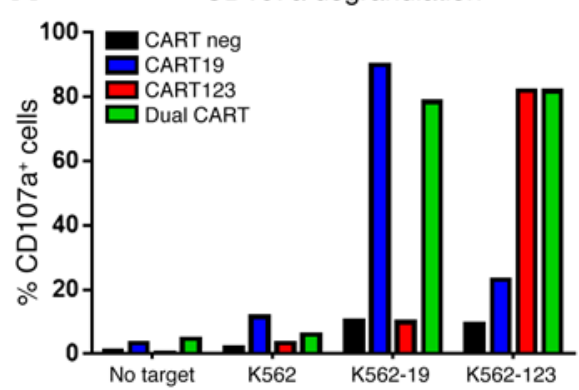

B

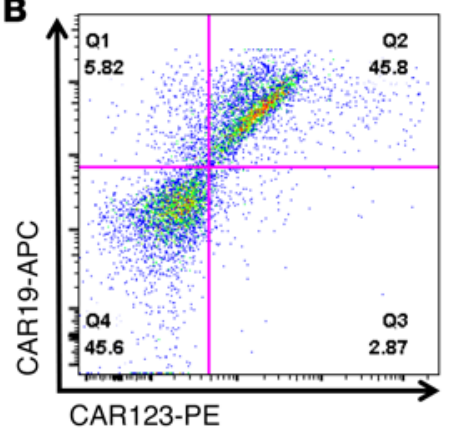

C

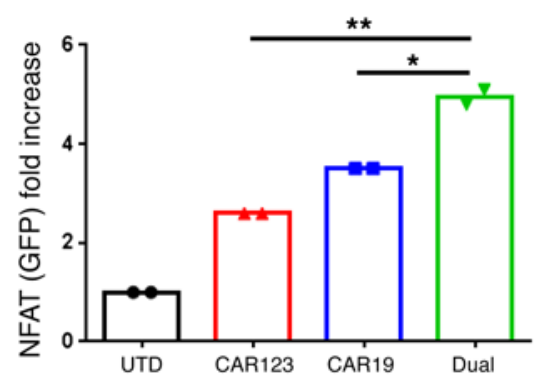

D

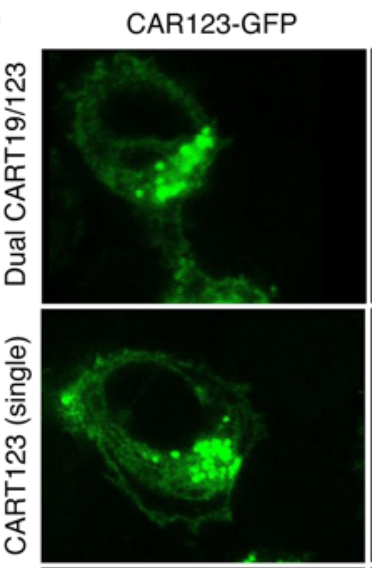

CAR19-mCherry

Cell trace violet (NALM6)

Overlay
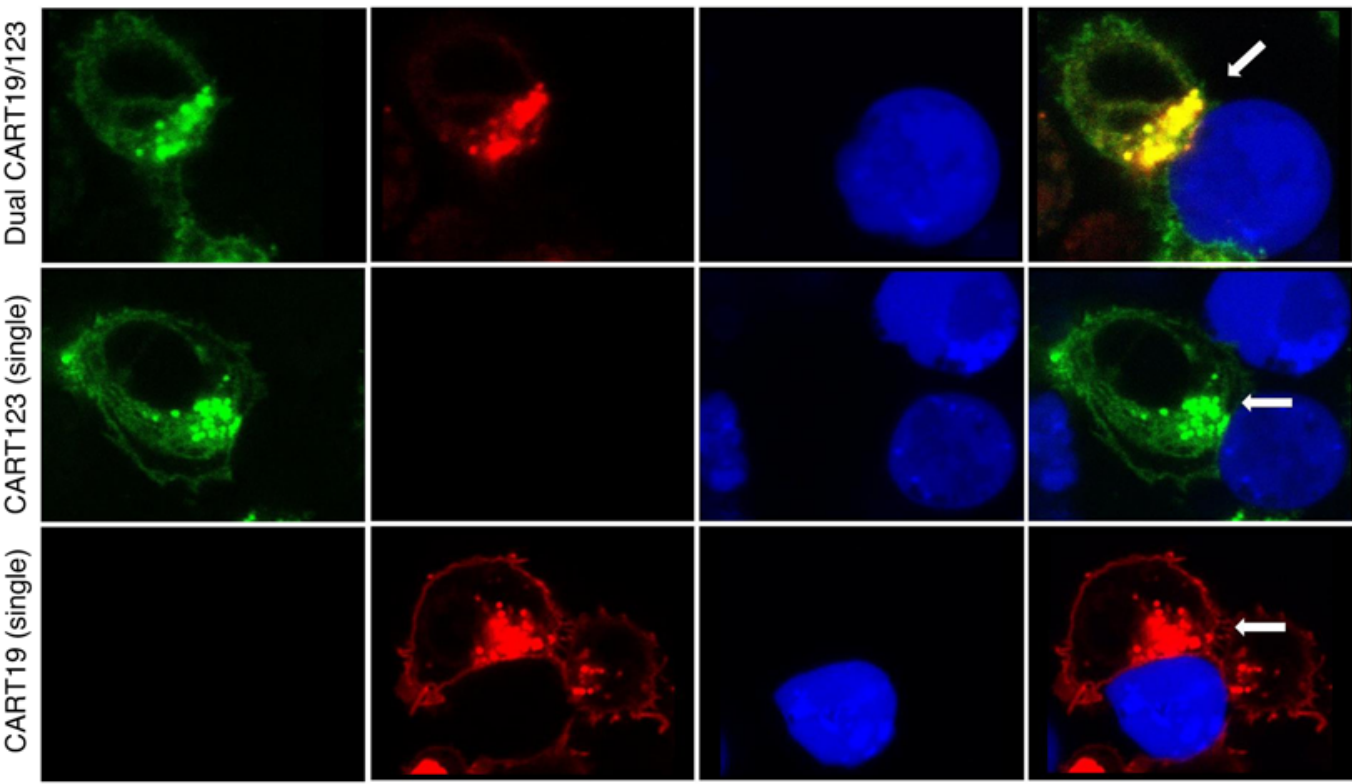

Figure 5. Potent activation and dual-specific immune synapse formation of dual CART19/123 cells. (A) Dual CART19/123 cells showed CD107a degranulation when cocultured for 6 hours with CD19-positive $\mathrm{K} 562$ cell line or $C D 123^{+} \mathrm{K} 562$, while single CART19+ or CART123+ cells were only able to recognize CD19+ or CD123+ K562, respectively. (B) A bicistronic P2A plasmid carrying both CART19 and CART123 was used to generate lentivirus and transduce T cells. As shown in the dot plot, transduced T cell display combined expression of CART19 and CART123. (C) Dual and single CAR+ Jurkat NFAT reporter cell lines were generated and used to test NFAT activation dynamics when cocultured with CD19+CD123+ K562 cells. At 1 hour, dual CARTs showed significantly higher NFAT activation as compared with either single-positive CART (CART19 or CART123). (D) Confocal imaging of dual CART revealed that both CARs (CART19, red, CART123, green) are simultaneously engaged in the same immune synapse (yellow) with leukemic cells (NALM6, stained with CellTrace Violet). Panels $A-C$ are representative of 2 independent experiments. Magnification, $\times 63$ (oil immersion). Student's $t$ test was used to compare 2 groups; in analysis where multiple groups were compared, 1-way ANOVA was performed with Holm-Šidák correction for multiple comparisons. ${ }^{*} P<0.05$; ${ }^{* *} P<0.01$.

Tracker Orange or TRITC, red) in mice bearing CD19-positive primary or CD19-negative relapsed blasts (both blasts express GFP) and, approximately 24 hours after injection (experiment schematic, Supplemental Figure 5A), imaged these populations using intravital 2-photon microscopy of calvarial marrow. These studies showed that CART19 and CART123 trafficked to marrow spaces containing leukemia blasts and that upon CART cell recognition of cognate antigen, the $\mathrm{T}$ cells underwent motility arrest as previously described in other tumor and infectious models (43). Specifically, in mice engrafted with the baseline $\mathrm{CD} 19^{+} \mathrm{CD} 123^{+}$leukemia, $62.9 \%( \pm 3.8 \%)$ of CART19 and $81.1 \%( \pm 1.2 \%)$ of CART123 were found to be stalled with a rounded morphology adjacent to blasts, whereas in mice engrafted with the relapsed $\mathrm{CD} 19^{-} \mathrm{CD} 123^{+}$leukemia, only CART123 cells arrested next to tumor cells (CART123 $80.9 \% \pm 5.1 \%$ vs. CART19 $12.4 \% \pm 2.2 \%, P<0.01$ ) (Supplemental
Figure 5, B and C, and Supplemental Videos 1 and 2). These findings indicate that in CD19-negative relapsed ALL, only CART123 were able to establish productive synapses with the leukemia cells (GFP), whereas CART19 cells continued sampling and moving in the environment without recognizing the leukemia blasts.

The combination of CART123 and CART19 is able to prevent CD19-negative relapses. Having shown that CART123 can treat CART19-resistant CD19-negative relapses, we next sought to develop a combinatorial preemptive approach to treating active CD19-positive disease while simultaneously preventing antigenloss relapses. In order to test this hypothesis, we modeled the emerging clinical problem of B-ALL with a potential for CD19negative escape by injecting primary $\mathrm{CD}^{-} 9^{-}$(at time of relapse) and $\mathrm{CD}_{19^{+}}$disease (at baseline, from the same patient) together into NSG mice. Mice were then randomized to receive control T 
A
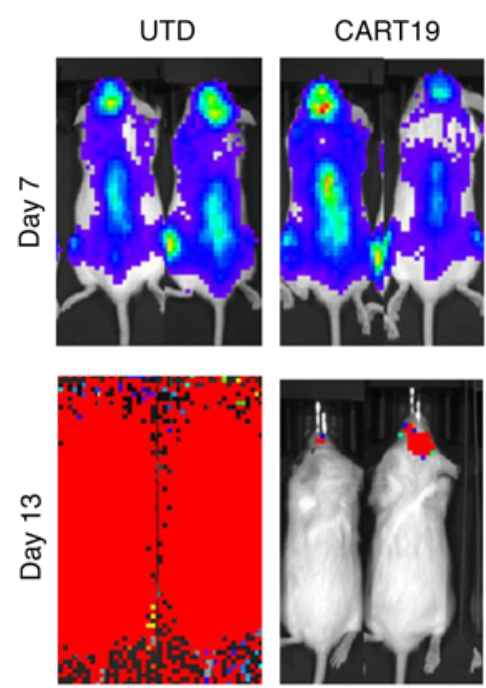

B

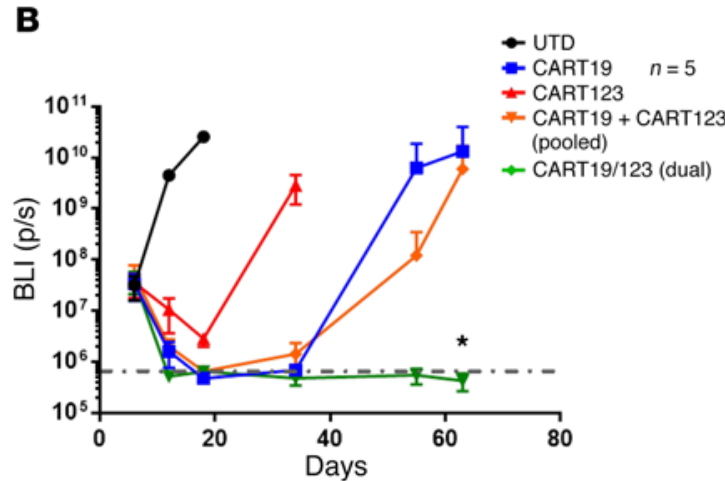

$\mathbf{E}$

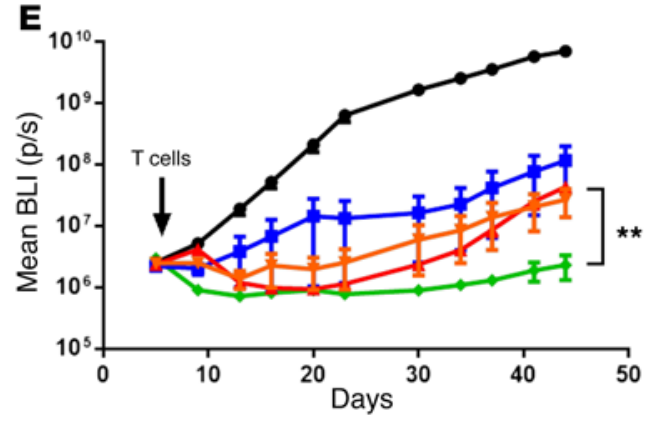

CART19+

CART123

(pooled)

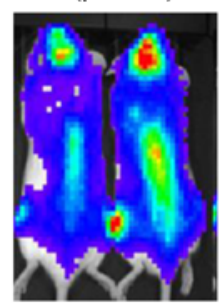

CART19/123

(dual)
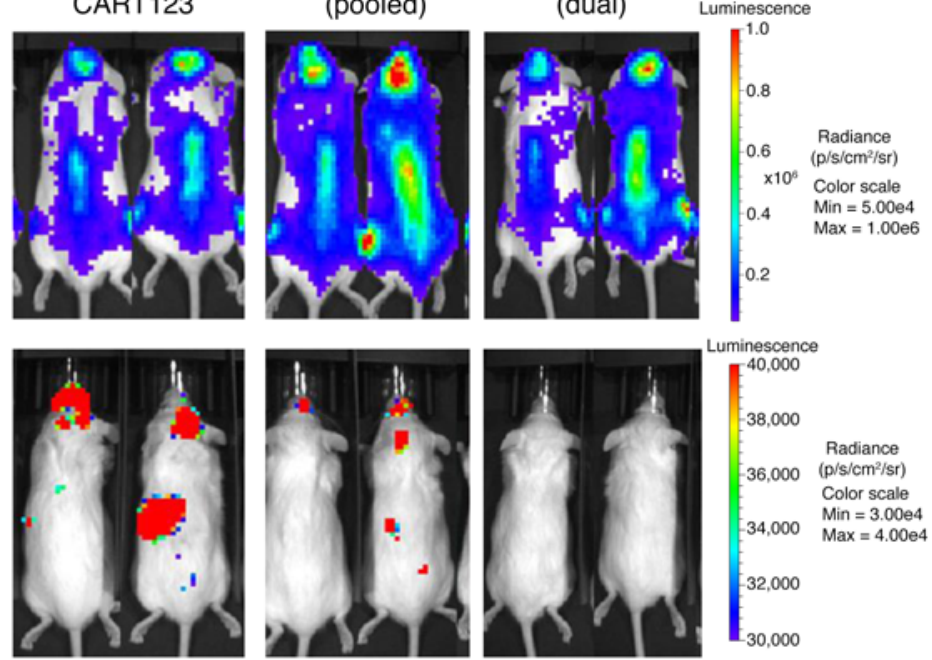

C

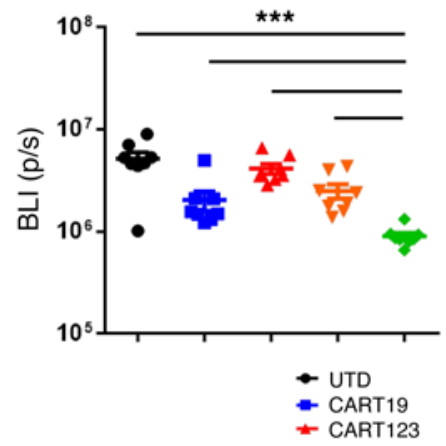

D

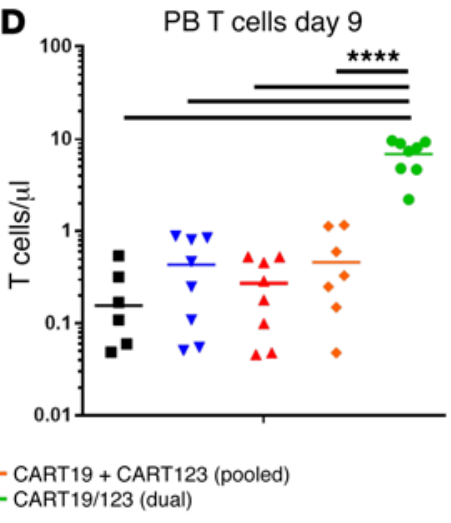

Figure 6. Dual CART19/123 cells are highly effective against B-ALL in vivo. (A) NSG mice were engrafted with a B-ALL cell line (NALM6, CBG+). At day 7, mice were randomized based on tumor burden (BLI) to receive control T cells (UTD), CART19, CART123, the 1:1 pooled combination of CART123 and CART19, or the dual CART19/123 (same total number of CAR+ cells). The tumor burden 6 days after T cell infusion (day 13) is shown in the graph: the deepest short-term antileukemia response is observed in the dual CART group. Monitoring of tumor burden (BLI) over time shows that only the dual CART19/123-treated mice have long-term CR $(P=0.02$ at day 63). (B) Bioluminescence imaging of mice receiving the different treatments at day 7 (before T cell infusion) and day 13 (6 days after T cell infusion). Only mice receiving dual CART19/123 reached a quick and deep complete response. All graphs representative of 2 independent experiments. (C) In a primary B-ALL xenograft model (patient UPN\#11), dual CART19/123 have a superior antileukemia activity (shown as reduced bioluminescence) as compared with pooled CART19 + CART123 at early time point (day 9), and this was correlated by a significantly higher T cell engraftment in the PB (D). (E) Tumor burden imaging (as mean bioluminescence) demonstrated a better antileukemia activity of dual CART19/123 as compared with pooled CART19 + CART123 or single CART. Panels B-E are representative of at least 2 independent experiments. Student's $t$ test was used to compare 2 groups; in analysis where multiple groups were compared, 1-way ANOVA was performed with Holm-Šidák correction for multiple comparisons. When multiple groups at multiple time points/ratios were compared, the Student's $t$ test or ANOVA for each time point/ ratio was used. ${ }^{*} P<0.05 ;{ }^{* *} P<0.01 ;{ }^{* * *} P<0.001 ;{ }^{* * *} P<0.0001$. 
cells (UTD), CART19, or a combination of CART19 and CART123, with the same total cell dose (Figure 4A). As shown in Figure 4B, mice treated with control $\mathrm{T}$ cells had progression of both leukemia clones (day 50, $P=$ NS) and mice treated with CART19 showed rapid progression mostly of the CD19-negative disease (day 50, $P=0.0079$ ). In contrast, mice treated with CART123 or with a combination of CART123 and CART19 showed clearance of the disease. Analysis of mice sacrificed at day 50 showed progression of both $\mathrm{CD}^{+} 9^{+}$and $\mathrm{CD} 19^{-}$disease in UTD-treated mice, while there was no evidence of residual leukemia in most of the mice of the combination CART cell group. In contrast, mice with progressive disease after CART19 monotherapy showed a CD19-negative phenotype (Figure 4C).

We hypothesized that dual-targeted $\mathrm{T}$ cells, i.e., expressing 2 CARs in the same T cells, as opposed to "pooled" CART, where 2 groups of CART express 2 different CARs, could provide a stronger antitumor effect than either CART alone. We transduced T cells with 2 lentiviruses, one carrying CART19 and the other CART123, in order to develop a CART capable of being activated by either CD19 or CD123. We could detect 4 differently transduced T cell subsets: CART19 and CART123 double-negative, CART19 single-positive, CART123 single-positive, and double-positive CART19/123 T cells (Supplemental Figure 6A). We sorted these 4 subsets and tested their functionality and specificity against K562 WT, K562 CD19+, or K562 CD123+. Figure 5A shows, as expected, that single CART cells responded to their specific target, while only the double-positive population was able to degranulate in the presence of both CD19- and CD123-expressing targets. We then generated a single bicistronic plasmid carrying both second-generation 41-BBz CARs, (CART19BBz-P2A-CART123BBz) that was shown to result in similar expression of both CARs (Figure 5B). Expression of 2 full second-generation CARs in the same plasmid was obtained utilizing codon-optimization strategies of the costimulatory domains (data not shown). In order to understand whether the presence of 2 CAR constructs in the same T cells could lead to better $\mathrm{T}$ cell activation, we generated WT, CART19+, $\mathrm{CART}^{2} 3^{+}$, and dual CART19/123+ Jurkat NFAT GFP reporter cell lines and analyzed NFAT activation by flow cytometry. Dual CART19/123 cells showed significantly higher NFAT activation at 1 hour compared with single $\mathrm{CAR}^{+} \mathrm{T}$ cells (Figure $5 \mathrm{C}$ ). Finally, we aimed to study the immune synapse of the dual CART. CAR19GFP and CAR123-mCherry fusion lentiviral constructs were generated and used to cotransduce T cells. Double-positive (dual) CART19/123 cells were isolated, cocultured with CellTrace Violet ${ }^{+}$NALM6 cells, and imaged by confocal microscopy. Interestingly, both CART19 (green) and CART123 (red) were engaged in the same immunological synapse (yellow) in dual CART19/123, as shown in Figure 5D.

Importantly, when tested in vivo, dual-stimulated CART19/ $123^{+}$cells exhibited higher efficacy against the B-ALL cell line NALM6 in comparison with an equivalent number of single-stimulated CART19 cells, single-stimulated CART123 cells, or pooled CART19 and CART123 cells at short $(P=0.006)$ and long term (Figure 6, A and B, and Supplemental Figure 6B), suggesting that a further increase in efficacy can be obtained by using a CAR that is triggered by 2 different antigens simultaneously. In order to test dual CART with a more clinically relevant model, we engrafted
NSG mice with primary B-ALL blasts (patient UPN\#09) that were transduced with a luciferase gene. Also in this model, dual CART19/123 exhibited higher antileukemia activity early (day 9) (Figure 6C), and this increased dual CART antitumor activity was correlated with a significantly higher peak $\mathrm{T}$ cell expansion in the PB (day 9), as shown in Figure 6D. In the long term, dual CART19/123 still maintained a significantly better antileukemia effect as compared with the other groups (Figure 6E).

\section{Discussion}

CD19-directed immunotherapies are changing the paradigm of treatment of relapsed refractory B-ALL. Patients with a previously dismal outcome now have a realistic potential to achieve a complete response and long-term disease remission (9). However, as shown under some circumstances for other types of leukemia treated with other forms of potent targeted therapy, leukemia cells are able to develop escape mechanisms that lead to resistance and relapse (44-46). In the case of CART19, 2 main patterns of relapses have been observed. Patients with early loss of CART19 through failure of persistence are at risk for relapse of the original clone; indeed, minimal residual disease analyses indicate that between 1 and 6 months of sustained CART activity may be required to completely eradicate malignancy $(6,41)$. In contrast, around $60 \%$ of relapses at our centers occur despite CART19 persistence and are characterized by the occurrence of a CD19-negative leukemia. The latter observation implicates potent selective pressure by CART19. Notably, CD19-negative relapses have also occurred after blinatumomab therapy, although these represent the minority of relapses after this arguably less persistent therapy (5). There are likely multiple potential mechanisms for the development of CD19-negative disease, while this phenomenon has been observed only anecdotally in animal models (D.M. Barrett, unpublished observations), and it is difficult to adequately recapitulate it in preclinical models. One of the mechanisms of antigen-loss relapse was recently reported by our group as alternative splicing of the CD19 gene that leads to the expression of variants lacking the domains required for recognition by CART19 (18). The goal of the current study was to define novel strategies to treat patients relapsing with antigen loss after CD19-directed therapies. Here, we describe the existence of rare CD19-negative cells in the malignant clone and propose that the selective pressure of CART19 therapy leads to their emergence as a clear example of immunoediting (41). We postulate that these CD19-negative cells are immature B-ALL precursors, as they exhibit the same cytogenetic aberration as the bulk leukemia and express the immature marker CD123, which has also been described as an LIC marker in AML (22). CD123 is, at the time of this writing, being studied as a target for immunotherapy in hematological neoplasms in 13 clinical trials (ClinicalTrials.gov as of 05/26/2016), including the CART123 trial for patients with $\mathrm{R} / \mathrm{R}$ myeloid leukemia that is, at the time of this writing, enrolling patients at the University of Pennsylvania. Genomic analysis of the clonal origins of relapsed ALL (47) indicates, as expected, that cells corresponding to the relapse clone are present as minor subpopulations at baseline, supporting our observation that minor CD19-negative clones are present at baseline, which is based on a combination of immunophenotyping and cytogenetics (47). Pre- 
vious work on ALL leukemic stem cells has implicated rare CD19negative populations $(25,48)$, suggesting that the transforming genetic event can occur in a committed progenitor (common lymphoid progenitor, pro-B cell or pre-B cell) where the predominant CD19-positive B cell phenotype occurs due to arrest at the pro- or pre-B cell stage (26). Here, we have shown for the first time, to our knowledge, that CD123 is expressed in the immunophenotypically-defined LIC in ALL, raising the possibility that targeting CD123 on LIC could promote ALL eradication. Regardless of its expression on a putative LIC, we confirmed CD123 to be highly expressed in the bulk leukemia population in the majority of primary B-ALL samples tested and, in particular, in those of our patients who relapsed with CD19-negative disease after CART19. Having demonstrated the presence of clonal leukemic cells in the $\mathrm{CD}^{-} 9^{-} \mathrm{CD} 123^{+}$population, we propose that targeting CD123 in combination with CD19 could enhance the likelihood of eradicating subclones that are otherwise prone to a selective advantage upon CART19 pressure (49). The concept that a combination of multiple targets is required to prevent antigen escapes for targeted immunotherapies is consistent with the early findings of chemotherapy treatment, where the use of a single agent led to frequent relapses and only a combined approached led to the current durable remissions in B-ALL.

To study the role of CART 123 in antigen-loss relapses, we developed a unique xenograft model of CD19-negative relapses from primary blasts derived from a B-ALL patient enrolled in the pediatric CTL019 trial of the University of Pennsylvania/Children's Hospital of Philadelphia. The baseline leukemia phenotype was $\mathrm{CD} 19^{+} \mathrm{CD} 123^{+}$, but at relapse after CART19 treatment, CD19 expression was lost. Using this model, we demonstrated that CART123 could eradicate the relapsed disease, and in combination with CART19, could prevent antigen-loss relapse. In addition, we showed, for what we believe is the first time using intravital imaging, that CART cells enter the marrow in under 24 hours after i.v. injection and their ability to arrest and interact with cognate antigen-bearing cells correlates with protective activity.

Furthermore, we showed the possibility of expressing 2 full CARs in a single $\mathrm{T}$ cell and that a dual signaling CART123/ CART19 was more effective than either CART alone or a pool of both CARTs. The concept of using more than one antigen to target cancer is actively pursued in the scientific community either using antibodies for leukemia (50) or CARs for solid tumors (51-57). We used 2 approaches: 2 separate lentiviral vectors and 2 separate $\mathrm{T}$ cell expansions to generate the pooled CART19/123 and, differently from previous studies, a single bicistronic vector encoding for 2 separate CARs and a single $\mathrm{T}$ cell expansion for our dual CART. The use of a bicistronic vector as opposed to 2 viruses and 2 transductions facilitated clinical translation and led to a 1:1 stoichiometric expression of the different CARs, as we have shown, rather than necessitating a cumbersome sorting step. To our knowledge, this is the first demonstration of a dual CART combination in a clinically relevant, antigen-loss model with important future clinical translation.

Our group has previously shown the preclinical efficacy of antiCD123 chimeric antigen receptor for the treatment of AML (38). We found that, along with strong antileukemia activity, CART123 can cause hematopoietic toxicity. However, our center and mul- tiple others are, at the time of this writing, evaluating CD123 as a viable antigen for AML treatment. A recent abstract reported on a single patient treated with lentivirally transduced CART123 and showed the feasibility of this approach (58). If hemopoietic toxicity develops in clinical trials, a possible path to clinical translation of the present preclinical study could include CART123 depletion (59) and a rescue allogeneic stem cell transplantation as is, at the time of this writing, practiced in several of the centers carrying out CART19 trials. Therefore, we have demonstrated a possible therapeutic strategy for dealing with the emerging clinical problem of antigen-loss relapses after potent targeted immunotherapies using the CART19 model and clinical samples from patients in our CTL019 trials. We showed, surprisingly, that dual CAR-expressing CART cells are more potent than single CAR expressing, correlating with enhanced early activation and augmented in vivo expansion. Additional studies will be necessary to further elucidate the complete mechanisms for this observation.

In summary, we described here an effective strategy for the treatment of B-ALL by targeting CD123, a stem cell marker expressed in CD19-negative blasts at baseline and relapse. This approach will be translatable in the future by combining dual CART19/123 upfront to prevent antigen loss relapses or by using CART123 to treat patients that develop CD19 relapses after CART19 administration.

\section{Methods}

Cell lines and primary samples. Cell lines were originally obtained from ATCC (K-562) or DSMZ (MOLM-14 and NALM6). All cell lines were tested for the presence of mycoplasma contamination (MycoAlert Mycoplasma Detection Kit, LT07-318, Lonza). For some experiments, cell lines were transduced with luciferase (firefly/EGFP, CBG/ EGFP, or CBR/EGFP) and then sorted to obtain a greater than $99 \%$ positive population. The K-562 cell line was also transduced with truncated CD19 or truncated CD123 to obtain cell lines expressing neither of them, only CD19, or only CD123. The Jurkat GFP-NFAT reporter cell line was available in C.H. June's laboratory and was originally obtained from System Biosciences Inc. (TR850A-1). It was transduced with CART19, CART123, and dual CART19/123 lentiviruses. MOLM-14 and K562 were used as controls, as indicated in the relevant figures. The cell lines were maintained in culture with RPMI 1640 (Gibco, LifeTechnologies, 11875-085) supplemented with 10\% FBS (Gemini, 100-106) and $50 \mathrm{UI} / \mathrm{ml}$ penicillin/streptomycin (Gibco, LifeTechnologies, 15070-063). Deidentified primary human ALL or normal donor BM and $\mathrm{PB}$ specimens were obtained from the clinical practices of University of Pennsylvania/Children's Hospital of Philadelphia under an IRB protocol or purchased from the Stem Cells and Xenograft Core of the University of Pennsylvania or from research samples of the current CTL019 clinical trials (Translation and Correlative Study Laboratory at the University of Pennsylvania). For all functional studies, primary cells were thawed at least 12 hours before experiments and rested at $37^{\circ} \mathrm{C}$.

In vivo expansion of primary $B-A L L$ blasts. Please see our previous descriptions of the establishment and maintenance of primary ALL and the 2 color luciferase imaging methods as described (42).

FISH. FISH analysis and immunohistochemistry were performed according to the standard method and as described (60). In brief, harvested ALL cells were suspended in fixative (acetic acid and metha- 
nol), deposited on the slides, and left to dry. Table 1 indicates the specific probes used for each sample. The appropriate probe to detect the known cytogenetic abnormality (dual color/dual fusion, break apart, or locus specific) probes (Abbott Molecular) were mixed with the hybridization buffer and applied on the slides. The slides were coverslipped, sealed, and left inside the ThermoBrite chamber for denaturation of the probe and target chromosomal DNA at $75^{\circ} \mathrm{C}$ for 10 minutes, followed by hybridization at $37^{\circ} \mathrm{C}$ for 16 hours. After removal of the sealant and the coverslip, slides were washed twice, blotted, dried, and counterstained with DAPI. Slides were examined under a fluorescent microscope, with a minimum of 200 (or 100 if less than 200) nuclei evaluated in each specimen.

Generation of CAR constructs and CART cells. The murine anti-CD19 chimeric antigen receptor (CD8 hinge, 4-1BB costimulatory domain, and CD3- $\zeta$ signaling domain) was generated as previously described $(61,62)$. This is the same construct used, at the time of this writing, in the CTL019 clinical trials at the University of Pennsylvania. For CART123, we used a scFv anti-CD123 (clone 32716) and the same backbone construct of CART19 (38). Production of CART cells was performed as previously described (38). Normal donor CD4 and CD8 T cells or peripheral blood mononuclear cells (PBMCs) were obtained from the Human Immunology Core of the University of Pennsylvania. Prior to all experiments, $\mathrm{T}$ cells were thawed and rested overnight at $37^{\circ} \mathrm{C}$.

Multiparametric flow cytometry. Flow cytometry was performed as previously described (63). Anti-human antibodies were purchased from BioLegend, eBioscience, or BD (please refer to Supplemental Table 1). For cell number quantitation, Countbright (Invitrogen) beads were used according to the manufacturer's instructions. In all analyses, the population of interest was gated based on forward- versus side-scatter characteristics followed by singlet gating, and live cells were gated using Live Dead Fixable Aqua (Invitrogen). Time gating was included for quality control. Surface expression of CART19 was detected as previously described, using an anti-idiotype antibody provided by L. Cooper (MD Anderson Cancer Center, Houston, Texas, USA) or using APC-conjugated CD19-Fc/His protein (64). Detection of CART123 was performed using goat anti-mouse antibody (Jackson Laboratories), CD123-Fc/His (Sino Biologicals) and anti-His-APC (R\&D), PE (AbCam), or directly PE-conjugated CD123 protein. Flow cytometry was performed on a 4-laser Fortessa-LSR II cytometer (BD) and analyzed with FlowJo X 10.0.7r2 (Tree Star).

In vitro $T$ cell effector function assays. Degranulation, CFSE proliferation, cytotoxicity assays, and cytokine measurements were performed as previously described $(38,64)$.

Animal experiments. In vivo experiments were performed as previously described (63). Schemas of the utilized xenograft models are discussed in detail in the relevant figure legends. NSG mice originally obtained from Jackson Laboratories were purchased from the Stem Cell and Xenograft Core of the University of Pennsylvania. Cells (leukemia cell lines or T cells) were injected in 100 to 200 $\mu \mathrm{l}$ of PBS at the indicated concentration into the tail veins of mice. Bioluminescent imaging was performed using a Xenogen IVIS-200 Spectrum camera and analyzed with LivingImage software v. 4.3.1 (Caliper LifeSciences). Animals were euthanized at the end of the experiment or when they met prespecified end points according to the IACUC protocols.

Multiphoton microscopy. Mice were anesthetized and maintained at core temperatures of $37^{\circ} \mathrm{C}$. BM was imaged after removing the scalp and immobilizing the skull. Imaging was performed using a Leica SP5 2-photon microscope system (Leica Microsystems) equipped with a picosecond laser (Coherent). Each imaging acquisition lasted 20 minutes followed by an assessment of mouse sedation. CellTrace Violet, GFP, and CellTrace Orange (or TRITC) were excited using a laser light of $850 \mathrm{~nm}$. Images were obtained using a $20 \times$ water-dipping lens. The resulting images were analyzed with Volocity software (PerkinElmer).

Confocal microscopy. Fluorescently tagged CART19-BB- $\zeta$ and CART123-BB- $\zeta$ lentiviral constructs were generated by introducing in-frame fusions of mCherry and GFP, respectively, using standard molecular cloning. Normal donor single or dual fluorescent CART cells were generated as described (65). T cells were cocultured with CellTrace Violet ${ }^{+}$NALM6 at a 1:1 effector-to-target (E:T) ratio for 2 hours on poly-D-lysine coated glass coverslips. Cells were washed, fixed with $4 \%$ paraformaldehyde, mounted onto slides with ProLong Diamond Antifade Mountant (Thermo Fisher), and imaged with a 63x oil immersion lens on a Leica TCS SP8 Laser Scanning confocal microscope (Leica Microsystems).

Statistics. All statistics were performed as indicated using GraphPad Prism 6 for Windows, version 6.05. Student's $t$ test was used to compare 2 groups; in analysis where multiple groups were compared, 1-way ANOVA was performed with Holm-Šidák correction for multiple comparisons. When multiple groups at multiple time points/ ratios were compared, Student's $t$ test (2-tailed) or ANOVA for each time point/ratio was used. Survival curves were compared using the log-rank test. Significance was defined as $P<0.05$.

Study approval. Human samples were obtained from the Stem Cell and Xenograft Core or the Human Immunology Core or were collected as research samples during the CTL019 clinical trials at the University of Pennsylvania. All patients or their parents gave informed consent. Animal experiments were performed according to a protocol (no. 803230) approved by the University of Pennsylvania IACUC that adheres to the NIH Guide for the Care and Use of Laboratory Animals (National Academies Press. 2011.).

\section{Author contributions}

MR, DMB, M Kalos, SG, and SAG formulated the initial ideas and planned the experiments. MR, DMB, SSK, and OS performed the experiments, analyzed the data, and contributed to the manuscript. JS and M Kozlowski participated in plasmid design. JJM, FN, and SFL provided the primary samples from the CTLO19 clinical trials. SFL designed and performed the Luminex assays. DAC and CAH performed the 2-photon intravital imaging. VA and JJDM performed the FISH analysis. M Klichinsky performed the confocal imaging. MR, DMB, and SG wrote the manuscript; SAG, DLP, and CHJ edited the manuscript. TJH, OS, and JP performed the animal experiments. All the authors reviewed and accepted the contents of the article.

\section{Acknowledgments}

The authors would like to thank Fang Chen and Natalka Kengle for the support with the Luminex cytokine assays. The authors would like to thank Charles "Hank" Pletcher and Paul Hallberg (Flow Cytometry Core, University of Pennsylvania) for help with the flow cytometry sorting of leukemia CD19-negative subsets. The chimeric antigen receptor used in this study was obtained under a materials transfer agreement from Dario Campana, Chihaya Imai, and the St. Jude Children's Research Hospital and was 
subsequently modified by cloning into a lentiviral vector and expressed with a eukaryotic promoter (62). This work was supported by grants from the University of Pennsylvania-Novartis Alliance (PI: CHJ and SG), NIH grant 5R01CA120409 (PI: CHJ), the EMD-Serono Cancer Immunotherapy Clinical Fellowship by the Society for Immunotherapy of Cancer (SITC) (PI: MR), the Bristol-Myers Squibb Oncology Fellowship in Clinical Cancer Research by the American Association for Cancer Research (AACR) (PI: MR), the Gabrielle's Angel Foundation (PI: MR), the SIES-AIL fellowship by the Italian Society for Experimental Hematology and the Italian Leukemia Association (PI: MR), and the St. Baldrick's Foundation Scholar Award and SU2C (PI: DMB and SAG). Imaging was performed at the University of Pennsyl- vania Small Animal Imaging Facility (SAIF) Optical/Bioluminescence Core, supported by NIH grant CA016520.

Address correspondence to: Saar Gill, Smilow Translational Research Center, 8-101, 3400 Civic Center Boulevard, Philadelphia, Pennsylvania 19104, USA. Phone: 215.573.4015; E-mail: saar.gill@uphs.upenn.edu. Or to: Stephan A. Grupp, Colket Translational Research Building, 3-006, 3501 Civic Center Boulevard, Philadelphia, Pennsylvania 19104, USA. Phone: 215.590.5476; E-mail: grupp@email.chop.edu.

M. Kalos' present address is Lilly Research Laboratories, Eli Lilly and Company, New York, New York, USA.
1. Bassan R, Hoelzer D. Modern therapy of acute lymphoblastic leukemia.JClin Oncol. 2011;29(5):532-543.

2. Fielding AK, et al. Outcome of 609 adults after relapse of acute lymphoblastic leukemia (ALL); an MRC UKALL12/ECOG 2993 study. Blood. 2007;109(3):944-950.

3. Tavernier E, et al. Outcome of treatment after first relapse in adults with acute lymphoblastic leukemia initially treated by the LALA- 94 trial. Leukemia. 2007;21(9):1907-1914.

4. Bhojwani D, Pui CH. Relapsed childhood acute lymphoblastic leukaemia. Lancet Oncol. 2013;14(6):e205-e217.

5. Topp MS, et al. Phase II trial of the anti-CD19 bispecific $\mathrm{T}$ cell-engager blinatumomab shows hematologic and molecular remissions in patients with relapsed or refractory B-precursor acute lymphoblastic leukemia. JClin Oncol. 2014;32(36):4134-4140.

6. Maude SL, et al. Chimeric antigen receptor T cells for sustained remissions in leukemia. N Engl JMed. 2014;371(16):1507-1517.

7. Davila ML, et al. Efficacy and toxicity management of 19-28z CAR T cell therapy in B cell acute lymphoblastic leukemia. Sci Transl Med. 2014;6(224):224ra25.

8. Lee DW, et al. T cells expressing CD19 chimeric antigen receptors for acute lymphoblastic leukaemia in children and young adults: a phase 1 doseescalation trial. Lancet. 2015;385(9967):517-528.

9. Ruella M, Gill S. How to train your T cell: genetically engineered chimeric antigen receptor $\mathrm{T}$ cells versus bispecific T-cell engagers to target CD19 in B acute lymphoblastic leukemia. Expert Opin Biol Ther. 2015;15(6):761-766.

10. Ruella M, Kalos M. Adoptive immunotherapy for cancer. Immunol Rev. 2014;257(1):14-38.

11. Turtle CJ, et al. CD19 CAR-T cells of defined $\mathrm{CD}^{+}: \mathrm{CD}^{+}$composition in adult B cell ALL patients. J Clin Invest. 2016;126(6):2123-2138.

12. Topp MS, et al. Long-term follow-up of hematologic relapse-free survival in a phase 2 study of blinatumomab in patients with MRD in B-lineage ALL. Blood. 2012;120(26):5185-5187.

13. Grupp SA, et al. Durable remissions in children with relapsed/refractory all treated with $\mathrm{T}$ cells engineered with a CD19-targeted chimeric antigen receptor (CTL019). Blood. 2015;126(23):681.

14. Chung EY, Psathas JN, Yu D, Li Y, Weiss MJ,
Thomas-Tikhonenko A. CD19 is a major B cell receptor-independent activator of MYCdriven B-lymphomagenesis. J Clin Invest. 2012;122(6):2257-2266.

15. Sultan I, Kraveka JM, Lazarchick J. CD19 negative precursor B acute lymphoblastic leukemia presenting with hypercalcemia. Pediatr Blood Cancer. 2004;43(1):66-69.

16. Moon H, Huh J, Cho MS, Chi H, Chung WS. A case of CD45-, CD19- precursor B cell acute lymphoblastic leukemia with an atypical morphology. Korean J Lab Med. 2007;27(4):253-256.

17. Niizuma H, Fujii K, Sato A, Fujiwara I, Takeyama J, Imaizumi M. PTHrP-independent hypercalcemia with increased proinflammatory cytokines and bone resorption in two children with CD19negative precursor B acute lymphoblastic leukemia. Pediatr Blood Cancer. 2007;49(7):990-993.

18. Sotillo E, et al. Convergence of acquired mutations and alternative splicing of CD19 enables resistance to CART-19 immunotherapy. Cancer Discov. 2015;5(12):1282-1295.

19. Hwang K, et al. Immunohistochemical analysis of CD123, CD56 and CD4 for the diagnosis of minimal bone marrow involvement by blastic plasmacytoid dendritic cell neoplasm. Histopathology. 2013;62(5):764-770.

20. Del Giudice I, et al. The diagnostic value of CD123 in B-cell disorders with hairy or villous lymphocytes. Haematologica. 2004;89(3):303-308.

21. Fromm JR. Flow cytometric analysis of CD123 is useful for immunophenotyping classical Hodgkin lymphoma. Cytometry B Clin Cytom. 2011;80(2):91-99.

22. Liu K, Zhu M, Huang Y, Wei S, Xie J, Xiao Y. CD123 and its potential clinical application in leukemias. Life Sci. 2015;122:59-64.

23. Hassanein NM, Alcancia F, Perkinson KR, Buckley PJ, Lagoo AS. Distinct expression patterns of CD123 and CD34 on normal bone marrow B-cell precursors ("hematogones") and B lymphoblastic leukemia blasts. Am J Clin Pathol. 2009;132(4):573-580.

24. Djokic M, Björklund E, Blennow E, Mazur J, Söderhäll S, Porwit A. Overexpression of CD123 correlates with the hyperdiploid genotype in acute lymphoblastic leukemia. Haematologica. 2009;94(7):1016-1019.

25. Cox CV, Evely RS, Oakhill A, Pamphilon DH, Goulden NJ, Blair A. Characterization of acute lymphoblastic leukemia progenitor cells. Blood. 2004;104(9):2919-2925.

26. Bernt KM, Armstrong SA. Leukemia stem cells and human acute lymphoblastic leukemia. Semin Hematol. 2009;46(1):33-38.

27. Muñoz L, et al. Interleukin-3 receptor alpha chain (CD123) is widely expressed in hematologic malignancies. Haematologica. 2001;86(12):1261-1269.

28. Jordan CT, et al. The interleukin-3 receptor alpha chain is a unique marker for human acute myelogenous leukemia stem cells. Leukemia. 2000;14(10):1777-1784.

29. Riccioni R, et al. Immunophenotypic features of acute myeloid leukemias overexpressing the interleukin 3 receptor alpha chain. Leuk Lymphoma. 2004;45(8):1511-1517.

30. Testa U, et al. Human acute stem cell leukemia with multilineage differentiation potential via cascade activation of growth factor receptors. Blood. 2002;99(12):4634-4637.

31. Angelot-Delettre F, et al. In vivo and in vitro sensitivity of blastic plasmacytoid dendritic cell neoplasm to SL-401, an interleukin-3 receptor targeted biologic agent. Haematologica. 2015;100(2):223-230.

32. Cohen KA, Liu TF, Cline JM, Wagner JD, Hall PD, Frankel AE. Safety evaluation of DT388IL3, a diphtheria toxin/interleukin 3 fusion protein, in the cynomolgus monkey. Cancer Immunol Immunother. 2005;54(8):799-806.

33. He SZ, et al. A Phase 1 study of the safety, pharmacokinetics and anti-leukemic activity of the antiCD123 monoclonal antibody CSL360 in relapsed, refractory or high-risk acute myeloid leukemia. Leuk Lymphoma. 2015;56(5):1406-1415.

34. Zereshkian A, Leyton JV, Cai Z, Bergstrom D, Weinfeld M, Reilly RM. The human polynucleotide kinase/phosphatase (hPNKP) inhibitor A12B4C3 radiosensitizes human myeloid leukemia cells to Auger electron-emitting anti-CD123 ${ }^{111}$ In-NLS-7G3 radioimmunoconjugates. $\mathrm{Nucl}$ Med Biol. 2014;41(5):377-383.

35. Kuo SR, Wong L, Liu JS. Engineering a CD123xCD3 bispecific scFv immunofusion for the treatment of leukemia and elimination of leukemia stem cells. Protein Eng Des Sel. 2012;25(10):561-569.

36. Chichili GR, et al. A CD3xCD123 bispecific DART for redirecting host $\mathrm{T}$ cells to myelogenous leukemia: preclinical activity and 
safety in nonhuman primates. Sci Transl Med. 2015;7(289):289ra82.

37. Fan D, et al. AntiCD3Fv fused to human interleukin-3 deletion variant redirected $T$ cells against human acute myeloid leukemic stem cells. J Hematol Oncol. 2015;8:18.

38. Gill S, et al. Preclinical targeting of human acute myeloid leukemia and myeloablation using chimeric antigen receptor-modified T cells. Blood. 2014;123(15):2343-2354.

39. Mardiros A, et al. T cells expressing CD123-specific chimeric antigen receptors exhibit specific cytolytic effector functions and antitumor effects against human acute myeloid leukemia. Blood. 2013;122(18):3138-3148.

40. Tettamanti S, Magnani CF, Biondi A, Biagi E. Acute myeloid leukemia and novel biological treatments: monoclonal antibodies and cellbased gene-modified immune effectors. Immunol Lett. 2013;155(1-2):43-46.

41. Grupp SA, et al. Chimeric antigen receptor-modified T cells for acute lymphoid leukemia. $N$ Engl J Med. 2013;368(16):1509-1518.

42. Barrett DM, et al. Noninvasive bioluminescent imaging of primary patient acute lymphoblastic leukemia: a strategy for preclinical modeling. Blood. 2011;118(15):e112-e117.

43. Harris TH, et al. Generalized Lévy walks and the role of chemokines in migration of effector CD8+ T cells. Nature. 2012;486(7404):545-548.

44. Vago L, et al. Loss of mismatched HLA in leukemia after stem-cell transplantation. $\mathrm{N}$ Engl J Med. 2009;361(5):478-488.

45. Chu SH, Small D. Mechanisms of resistance to FLT3 inhibitors. Drug Resist Updat. 2009;12(1-2):8-16.

46. Soverini S, et al. BCR-ABL kinase domain mutation analysis in chronic myeloid leukemia patients treated with tyrosine kinase inhibitors: recommendations from an expert panel on behalf of European LeukemiaNet. Blood. 2011;118(5):1208-1215.
47. Mullighan CG, et al. Genomic analysis of the clonal origins of relapsed acute lymphoblastic leukemia. Science. 2008;322(5906):1377-1380.

48. Castor A, et al. Distinct patterns of hematopoietic stem cell involvement in acute lymphoblastic leukemia. Nat Med. 2005;11(6):630-637.

49. Francis J, et al. CD19 expression in acute leukemia is not restricted to the cytogenetically aberrant populations. Leuk Lymphoma. 2013;54(7):1517-1520.

50. Kügler M, et al. A recombinant trispecific singlechain $\mathrm{Fv}$ derivative directed against $\mathrm{CD} 123$ and CD33 mediates effective elimination of acute myeloid leukaemia cells by dual targeting. $\mathrm{Br} \mathrm{J}$ Haematol. 2010;150(5):574-586.

51. Lanitis E, et al. Chimeric antigen receptor $\mathrm{T}$ cells with dissociated signaling domains exhibit focused antitumor activity with reduced potential for toxicity in vivo. Cancer Immunol Res. 2013;1(1):43-53.

52. Kloss CC, Condomines M, Cartellieri M, Bachmann M, Sadelain M. Combinatorial antigen recognition with balanced signaling promotes selective tumor eradication by engineered T cells. $\mathrm{Nat}$ Biotechnol. 2013;31(1):71-75.

53. Hegde $\mathrm{M}$, et al. Combinational targeting offsets antigen escape and enhances effector functions of adoptively transferred $\mathrm{T}$ cells in glioblastoma. Mol Ther. 2013;21(11):2087-2101.

54. Wilkie S, et al. Dual targeting of ErbB2 and MUC1 in breast cancer using chimeric antigen receptors engineered to provide complementary signaling. JClin Immunol. 2012;32(5):1059-1070.

55. Anurathapan U, et al. Kinetics of tumor destruction by chimeric antigen receptor-modified $\mathrm{T}$ cells. Mol Ther. 2014;22(3):623-633.

56. Fedorov VD, Themeli M, Sadelain M. PD-1and CTLA-4-based inhibitory chimeric antigen receptors (iCARs) divert off-target immunotherapy responses. Sci Transl Med. 2013;5(215):215ra172.
57. Zah E, Lin MY, Silva-Benedict A, Jensen MC, Chen YY. T cells expressing CD19/CD20 bispecific chimeric antigen receptors prevent antigen escape by malignant B cells. Cancer Immunol Res. 2016;4(6):498-508.

58. Luo Y, Chang L-J, Hu Y, Dong L, Wei G, Huang H. First-in-man CD123-specific chimeric antigen receptor-modified $\mathrm{T}$ cells for the treatment of refractory acute myeloid leukemia. Blood. 2015;126(23):3778.

59. Tasian SK, et al. Efficient termination of CD123redirected chimeric antigen receptor $\mathrm{T}$ cells for acute myeloid leukemia to mitigate toxicity. Blood. 2015;126(23):565.

60. Belaud-Rotureau MA, Parrens M, Dubus P, Garroste JC, de Mascarel A, Merlio JP. A comparative analysis of FISH, RT-PCR, PCR, and immunohistochemistry for the diagnosis of mantle cell lymphomas. Mod Pathol. 2002;15(5):517-525.

61. Milone MC, et al. Chimeric receptors containing CD137 signal transduction domains mediate enhanced survival of $\mathrm{T}$ cells and increased antileukemic efficacy in vivo. Mol Ther. 2009;17(8):1453-1464.

62. Imai C, et al. Chimeric receptors with $4-1 \mathrm{BB}$ signaling capacity provoke potent cytotoxicity against acute lymphoblastic leukemia. Leukemia. 2004;18(4):676-684.

63. Kenderian SS, et al. CD33-specific chimeric antigen receptor $\mathrm{T}$ cells exhibit potent preclinical activity against human acute myeloid leukemia. Leukemia. 2015;29(8):1637-1647.

64. Kalos M, et al. T cells with chimeric antigen receptors have potent antitumor effects and can establish memory in patients with advanced leukemia. Sci Transl Med. 2011;3(95):95ra73.

65. Ruella M, et al. The addition of the BTK inhibitor Ibrutinib to anti-CD19 chimeric antigen receptor T cells (CART19) improves responses against mantle cell lymphoma. Clin Cancer Res. 2016;22(11):2684-2696. 